\title{
EINIGE BEOBACHTUNGEN UBER DAS MESSEN DER BODENTEMPERATUR BEI ANWENDUNG VON THERMOELEMENTEN
}

\author{
TANeli JuUsela. \\ Institut für Pflanzenbau der Universität Helsinki. \\ Eingegangen am 4. VI. 1945 ,
}

Bei der Messung der Bodentemperatur werden im allgemeinen entweder Flüssigkeitsthermometer oder elektrische Messverfahren angewandt. Zur Bestimmung der Bodentemperatur sind besondere gekrümmte Quecksilberthermometer angefertigt worden, bei denen die Quecksilberkugel unter einem Winkel von $120^{\circ}$ zum Skalenteil liegt, und steht bei der Verwendung der Thermometer waagerecht im Erdboden. Derartige Flüssigkeitsthermometer entschprechen dennoch nicht den Forderungen, die die Messungen der Bodentemperatur an sie stellt (vgl. 5, S. 17-23 und 4, S. 5). So ist es u.a. beinahe unmöglich sie so zu befestigen, dass sich die Wärmeleitfähigkeit von der Erdoberfläche bis zum Messpunkt nicht veränderte und da der Skalenteil in der Richtung der Wärmeströmung durch die Erdschicht verläuft, kann auch die Luftströmung und das einsickernde Regenwasser Fehler verursachen. Ausserdem eignen sich die Flüssigkeitsthermometer nicht für Serienbeobachtungen und sind wegen ihrer Zerbrechlichkeit auch vom Wintergebrauch ausgeschlossen. Dessen ungeachtet werden jene wegen ihres billigen Preises und ihrer einfachen Handhabung noch heute sehr häufig angewandt (2, 7 und 10).

Der grosse Vorzug der elektrischen Messverfahren, der Wiederstandsthermometer und der Thermoelemente ist ihr geringer Umfang, wodurch sie die natürlichen Wärmeverhältnisse des Erdbodens kaum verändern, und auch mit grosser Empfindlichkeit auf die Temperaturveränderungen ihrer Umgebung reagieren, eignen sich ausserdem besonders für Serienbeobachtungen im Winter. Im Ver- 
gleich mit einander sind beide Messverfahren zur Feststellung der Bodentemperatur ungefähr gleich gut.

In Finnland hat KeRÄNEN (4, S. 1-34) schon im Jahre 1915 in Sodankylä Messungen der Bodentemperatur mit Thermoelementen ausgeführt und später haben u. a. Schmidt (6), Herr (1, S. 6-8) und Juusela (3, S. 99-119) die gleiche Messmethode benutzt.

Da bei der Messung von elektromotorischen Kräften Thermoelemente benutzt werden, kann die Messung z.B. so vollzogen werden,

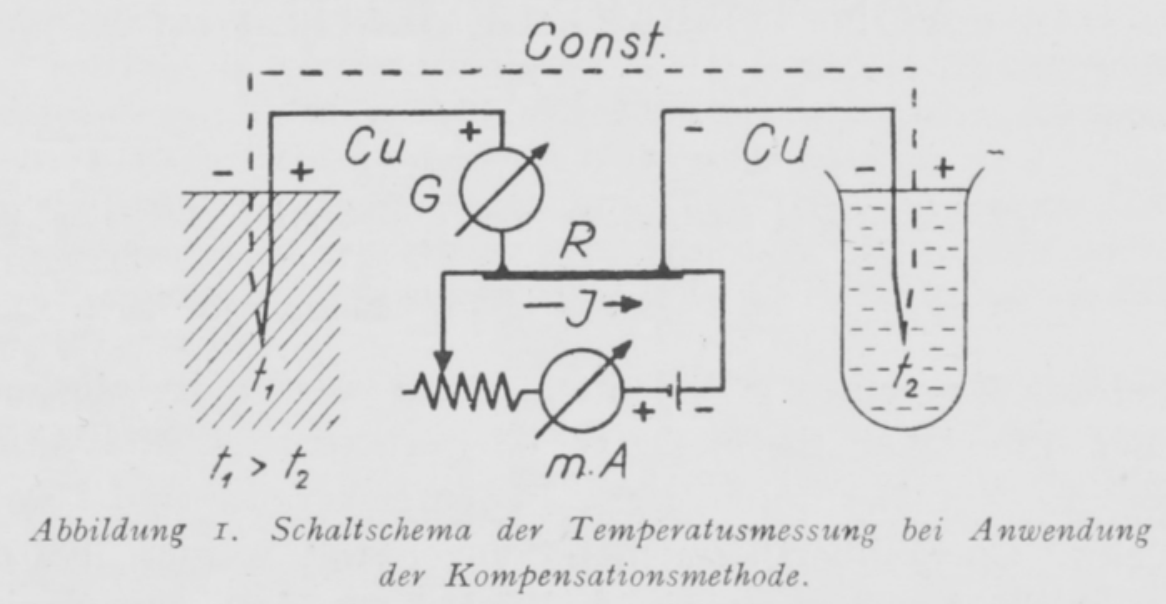

dass in den Thermoelementkreis ein hinreichend empfindlicher Galvanometer eingeschaltet wird, so dass zwischen der zu messenden Temperatur, der Temperatur des Vergleichselements und der Ablesung des Galvanometers eine annähernd lineare Gleichung herrscht. Weil bei einem derartigen Messsystem der totale Widerstand aus dem Galvanometerwiderstand nebst den Leitungswiderständen besteht, ist es angebracht die zu den verschiedenen Messungspunkten laufenden Leitungen aus so dickem Draht herzustellen, dass der Widerstand in den Beobachtungspunkten im Vergleich mit jenen der Teile der Messapparatur möglichst gering ist. Wenn die Temperatur der Messhütte nicht viele schwankt, so verursachen die übrigen ausserhalb des Messpunkts vorkommenden Temperaturschwankungen und die ungleiche Länge der Leitungen keine erheblichen Veränderungen im Gesamtwiderstand des Stromkreises, sodass die hierdurch entstandenen Fehler gering sind.

Der die Anwendung dieser einfachen Messmethode beeinträchtigende Fehler kann ganz beseitigt werden, indem die zu messende thermoelektrische Spannung mit Hilfe des elektrischen Stromes durch 
die zwischen den Polen eines bekannten Widerstands ergänzte Spannung kompensiert wird, wobei in dem Thermoelementkreis im Messungsmoment kein elektrischer Strom fliesst. (Abbildung 1, vgl. ebenfalls 9, S. 16.)

Auf diese Weise wurde u.a. in Backas i. d. J. 1940—42 bei den Temperaturmessungen verfahren. Die damals angewandte Messapparatur und deren eingehendes Schaltschema ist in meiner früheren Abhandlung (3, S. 99-119) angeführt. In diesem Zusammenhang werden einige auf die Zuverlässigkeit der Messergebnisse wirkende Faktoren näher verdeutlicht.

\section{Die Eichung der Temperaturmessapparatur.}

Ausser den im physikalischen Laboratorium der Universität ausgeführten Kontrollmessungen und Eichungen, wurde die Grundeichung der Messapparatur erst nachdem die Apparatur an Ort und Stelle auf dem Felde in Backas angebracht war vollzogen.

Bei der Eichung durfte die Temperatur der Vergleichslötstelle in der mit Paraffinöl gefüllten, geschlossenen Thermosflasche ganz ebenso wie bei den gewöhnlichen täglichen Temperaturbeobachtungen den äusseren Temperaturschwankungen der ungeheizten Beobachtungshütte frei folgen. Die Temperatur der Thermosflasche veränderte sich bei diesem Verfahren um ca. $0.2-0.6^{\circ}$ in der Stunde. Das nach Art der Vergleichslötstelle gebaute Eichungslötstelle wurde in ein ungefähr 501 fassendes, mit Wasser gefülltes Gefäss gestellt, in dem das Wasser andauernd während der ganzen Zeit der Eichung umgerührt wurde. Ausserdem wurden die Quecksilberthermometer an die sowohl die Vergleichs-, wie auch die Eichungselemente befestigt waren zwecks Verkleinerung der Korrektionsfehler so tief in der Flüssigkeit gehalten, wie es wegen ihrer Ablesung möglich war.

Das Eichgefäss wurde zunächst mit kaltem Wasser gefüllt, und seine Temperatur wurde immer nach der Beobachtung in der Weise verändert, dass ein Teil des Wassers entfernt und dafür unter stetigem Umrühren warmes Wasser zugegossen wurde. So wurde verfahren, bis der grösste Temperaturunterschied, der mit der Messapparatur ermittelt werden konnte, erreicht war, und hiernach wurde damit begonnen, die Temperatur des Eichgefässes auf dieselbe Weise herabzusetzen. Die Eichmessungen wurden den ganzen Tag hindurch mit etwa halbstündigen Intervallen ausgeführt, nachdem 
jedesmal festgestellt war, dass die Temperatur des Quecksilberthermometers, woran die Lötstelle des Thermometers befestigt war, beinahe unverändert blieb.

Zur Messung verschiedengrosser Temperaturunterschiede standen zwei Messbereiche zur Verfügung, wovon das erste (Kopplung 1) bei einem Temperaturunterschied unter $12^{\circ}$ und das zweite (Kopplung 2) bei $12-24^{\circ}$ angewandt wurde. Der Ausschlag des Amperemeters wurde bei beiden Kopplungen beobachtet, und die Anzeigen der beiden mit 1/10 -Einteilung versehenen Quecksilberthermometer wurden schätzungsweise mit 1/100 Grad Genauigkeit abgelesen. Um Beobachtungsfehler zu vermeiden, erfolgte die Ablesung der Quecksilberthermometer und des Amperemeters abwechselnd zweimal.

Bei der Prüfung der Eichergebnisse wurde gefunden, dass bei Anwendung der Kopplung 1, wo 50 Skalenteilen des Amperemeters ein Temperaturunterschied von $12.16^{\circ}$ entsprach, zwischen diesem und der Ablesung des Amperemeters die lineare Gleichung

$$
1=\text { at }
$$

herrschte, worin $\mathrm{t}=$ Temperaturunterschied, $1=$ Ablesung des Amperemeters und a ein Koeffizient, der die durch einen Temperaturunterschied von einem Grad verursachte Ablesung angibt. Als dessen Wert wurde $\frac{5000}{1216} \sim 4.112$ erhalten (vgl. 6, S. 412, 4, S. 23 und 1, S. 6-7). Die aus den Eichergebnissen gewonnenen Punkte fielen entweder auf diese Gerade oder lokalisierten sich beiderseits von ihr (Tabelle 1).

Der Mittelwert aus den absoluten Werten der Abweichungen der Eichergebnisse betrug $0.03^{\circ}$, während die grössten Abweichungen $+0.05^{\circ}$ und $-0.06^{\circ}$ waren. Die Abweichungen waren also auch in den verschiedenen Skalenbereichen des Amperemeters verhältnismässig gleichartig.

Wenn die Kopplung 2 benutzt wurde, wobei 50 Skalenteilen des Amperemeters ein Temperaturunterschied von $23.71^{\circ}$ entsprach, war die graphische Darstellung der Eichergebnisse schon deutlich eine gekrümmte Linie. Gewöhnlich wird sie in Form einer Gleichung zweiten Grades

$$
1=a t+b^{2}
$$

ausgedrückt, worin $1=$ Ablesung des Amperemeters, $\mathrm{t}=$ Temperaturunterschied und a und b Koeffizienten, deren Werte auf Grund der 
Tabelle I. Eichergebnisse bei Anwendung der Kopplung I

\begin{tabular}{|c|c|c|c|}
\hline $\begin{array}{c}\text { Ausschlag des } \\
\text { Amperemeters }\end{array}$ & $\begin{array}{c}\text { Berechnetes } \\
\text { Temperaturunterschied }\end{array}$ & $\begin{array}{c}\text { Beobachtetes } \\
\text { Temperaturunterschied }\end{array}$ & $\begin{array}{c}\text { Abweichungen } \\
\text { oder }\end{array}$ \\
\hline & $0.00^{\circ}$ & $0.05^{\circ}$ & $+0.05^{\circ}$ \\
0.0 & 0.19 & 0.14 & -0.05 \\
3.1 & 0.75 & 0.75 & \pm 0.00 \\
5.3 & 1.29 & 1.29 & \pm 0.00 \\
9.4 & 2.28 & 2.24 & -0.04 \\
11.7 & 2.85 & 2.83 & -0.02 \\
17.8 & 4.32 & 4.37 & +0.05 \\
22.9 & 5.57 & 5.54 & -0.03 \\
23.7 & 5.76 & 5.70 & -0.06 \\
28.3 & 6.88 & 6.89 & +0.01 \\
33.7 & 8.20 & 8.15 & -0.05 \\
37.8 & 9.19 & 9.20 & +0.01 \\
42.2 & 10.26 & 10.28 & +0.02 \\
49.2 & 11.96 & 11.94 & -0.02 \\
& & & \\
\hline
\end{tabular}

Eichergebnisse mittels der Gaussschen Methode der kleinsten Quadratsumme berechnet werden können (4, S. 24). Da sich jedoch die Eichtafel auch durch ein einfacheres Verfahren mit hinreichender Genauigkeit bestimmen lässt, wurde ihre Ausrechnung folgendermassen durchgeführt.

Die graphische Darstellung wurde als fünfteilige gebrochene Linie behandelt, deren Knickpunkte bei den Ablesungen 10, 20, 30 und 40 des Skalenbereichs des Amperemeters gewählt wurden. Nach mehreren Versuchen ergab sich schliesslich eine gebrochene Linie, deren geradlinigen Teilen folgende Temperaturunterschiede entsprechen:

Skalenbereich des Amperemeters

$$
\begin{array}{r}
0-10 \\
10-20 \\
20-30 \\
30-40 \\
40-50
\end{array}
$$

Temperaturunterschied

$$
\begin{aligned}
& 4.80^{\circ} \\
& 4.79^{\circ} \\
& 4.77^{\circ} \\
& 4.71^{\circ} \\
& 4.64^{\circ}
\end{aligned}
$$

Von den nach dieser gebrochenen Linie berechneten Werten wichen die Eichpunkte gemäss der Tabelle $2 \mathrm{ab}$.

Bei Anwendung der Kopplung 2 war der Mittelwert aus den absoluten Werten der Abweichungen der Eichergebnisse im ganzen Skalen- 
Tabelle 2. Eichergebnisse bei Anwendung der Kopplung 2.

\begin{tabular}{|c|c|c|c|}
\hline $\begin{array}{l}\text { Ausschlag des } \\
\text { Amperemeters }\end{array}$ & $\begin{array}{l}\text { Berechneter Tempe- } \\
\text { raturunterschied }\end{array}$ & $\begin{array}{l}\text { Beobachteter Tempe- } \\
\text { raturunterschied }\end{array}$ & $\begin{array}{l}\text { Abweichungen } \\
+ \text { oder - }\end{array}$ \\
\hline 0.4 & $0.19^{\circ}$ & $0.14^{\circ}$ & $-0.05^{\circ}$ \\
\hline 1.2 & 0.58 & 0.62 & +0.04 \\
\hline 1.6 & 0.77 & 0.75 & -0.02 \\
\hline 2.7 & 1.30 & 1.28 & -0.02 \\
\hline 4.8 & 2.30 & 2.23 & -0.07 \\
\hline 5.0 & 2.78 & 2.81 & +0.03 \\
\hline 9.2 & 4.42 & 4.36 & -0.06 \\
\hline \multicolumn{3}{|c|}{ Mittelwert aus den absoluten Werten der Abweichungen } & 0.04 \\
\hline 14.3 & 6.86 & 6.88 & +0.02 \\
\hline 17.1 & 8.20 & 8.15 & -0.05 \\
\hline 19.2 & 9.21 & 9.20 & -0.01 \\
\hline \multicolumn{3}{|c|}{ Mittelwert aus den absoluten Werten der Abweichungen } & n $\quad 0.03$ \\
\hline 21.5 & 10.31 & 10.27 & +0.04 \\
\hline 23.2 & 11.12 & 11.12 & 0.00 \\
\hline 24.2 & 11.60 & 10.64 & -0.04 \\
\hline 24.6 & 11.79 & 11.86 & -0.07 \\
\hline 25.1 & 12.03 & 11.94 & +0.09 \\
\hline 25.2 & 12.08 & 12.12 & -0.04 \\
\hline 27.8 & 13.31 & 13.34 & -0.03 \\
\hline \multicolumn{3}{|c|}{ Mittelwert aus den absoluten Werten der Abweichungen } & 0.04 \\
\hline 30.8 & 14.74 & 14.72 & -0.02 \\
\hline 31.2 & 14.92 & 14.94 & +0.02 \\
\hline 32.6 & 15.58 & 15.56 & -0.02 \\
\hline 33.4 & 15.96 & 15.89 & -0.07 \\
\hline 36.6 & 17.47 & 17.45 & -0.02 \\
\hline 36.7 & 17.52 & 17.52 & \pm 0.00 \\
\hline 37.3 & 17.80 & 17.88 & +0.08 \\
\hline 38.6 & 18.41 & 18.43 & +0.02 \\
\hline \multicolumn{3}{|c|}{ Mittelwert aus den absoluten Werten der Abweichungen } & 0.03 \\
\hline 41.8 & 19.91 & 19.84 & -0.07 \\
\hline 42.9 & 20.42 & 20.38 & -0.04 \\
\hline 44.5 & 21.16 & 21.21 & +0.05 \\
\hline 45.2 & 21.48 & 21.48 & \pm 0.00 \\
\hline 46.3 & 21.99 & 22.00 & +0.01 \\
\hline 47.2 & 22.41 & 22.39 & -0.02 \\
\hline 48.2 & 22.87 & 22.87 & \pm 0.00 \\
\hline 49.1 & 23.29 & 23.29 & \pm 0.00 \\
\hline 49.9 & 23.66 & 23.65 & -0.01 \\
\hline \multicolumn{3}{|c|}{ Mittelwert aus den absoluten Werten der Abweichungen } & en $\quad 0.02$ \\
\hline
\end{tabular}


bere.ch $0.03^{\circ}$, während die grössten Abweichungen $-0.09^{\circ}$ und $+0.08^{\circ}$ betrugen, auch waren die Abweichungen in den verschiedenen Skalenbereichen verhältnismässig gleichartig.

Ausser der vor Béginn der Messungen ausgeführten Grundeichung müssen während der Messperiode andauernd von Zeit zu Zeit Kontrolleichungen ausgeführt und der Wiederstand der Leitungen gemessen werden um die Messgenauigkeit zu sichern und um mögliche Lecke festzustellen und aufzufinden (3, S. 116-118).

Wie die oben angeführte Eichung der Messaparatur zeigt, erzielt man sogar mit Verwendung eines so einfachen Verfahrens eine vollkommen genügende Genauigkeit, denn wegen der Unhomogenität des Bodens kann die Anführung der Messergebnisse mit grösserer Genauigkeit als $0,1^{\circ}$ nicht für geeignet betrachtet werden.

\section{Über die Wirkung der Abtrennung des Bleimantels am Thermoelementkabel.}

Da bei den in Backas ausgeführten Temperaturmessungen des Erdbodens mit Bleimänteln versehene Kabel verwendet wurden und da die Wärmeleitfähigkeit des Bleis mehr als 10 mal grösser als die des Erdbodens ist, wurden um hierdurch verursachte Fehler zu vermeiden, wenn die Kabel durch solche Bodenschichten liefen, deren Temperatur erheblich von jener des Messpunktes abwich, einige Vorsichtsmassnahmen getroffen (vgl. 3, S. 112).

Um den auf diese Weise entstandenen Fehler, der schon in den vorberei enden Versuchen festgestellt wurde und mehrere ZehntelGrade ausmachen konnte, zu reduzieren, wurde der Bleimantel der Kabel auf einer Strecke von etwa $5 \mathrm{~cm} 70-75 \mathrm{~cm}$ von der Lötstelle entfernt ganz weggenommen. Um Lecke zu vermeiden, wurde die Schnittstelle durch Spezialleim und durch einen mit Binden versehenen Gummischlauch geschützt (Abb. 2).

Ausserdem wurde die Schnittstelle mit einer $20 \mathrm{~cm}$ langen $\mathrm{Ba}-$ kelitröhre, die mit Parafin vollgegossen wurde, geschützt.

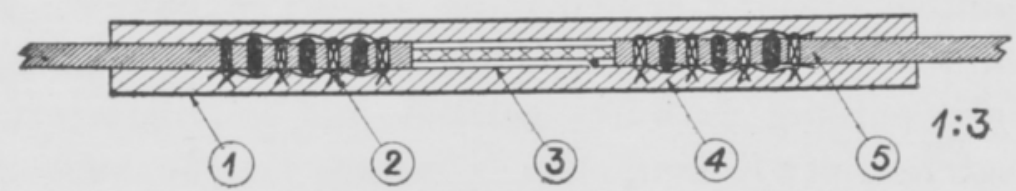

Abbildung. 2. Durchtrennung des Bleimantels an dem Thermoelementkabel. $I=$ Bakelitröhre, $2=$ Bindungen, $3=$ Gummischlauch, $4=$ Parafinfüllung, $5=$ Kabel . 
Um die Wirkung der Abtrennung des Bleimantels aufzuklären, wurde eine Versuchsserie derart angeordnet, dass in 30,60 und 90 $\mathrm{cm}$ Tiefe unter der Erdoberfläche in der gleichen waagerechten Ebene parallel $10 \mathrm{~cm}$ voneinander drei Thermoelementkabel angebracht wurden. An den äusseren dieser Kabel war der Bleimantel abgeschnitten, aber an dem mittleren setzte er sich ununterbrochen von der Thermoelementlötställe bis zur Verbindungsstelle der Kabel fort (Abbildung 3).
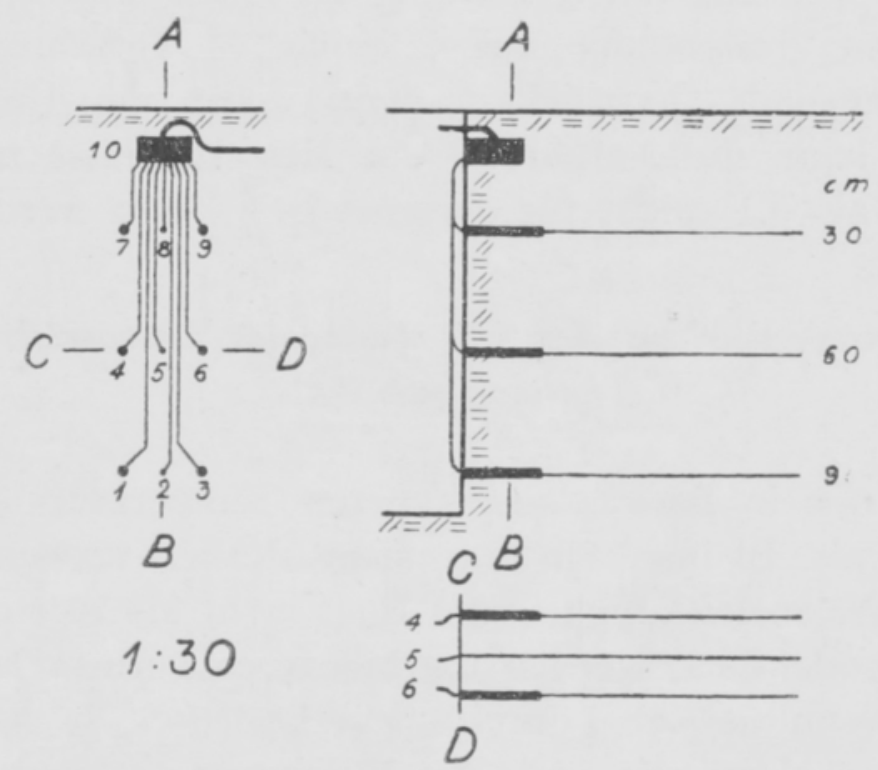

Abbildung 3. Temperaturmessungsversuchsserie zur Untersuchung der Wirkung der Durchtrennung des Bleimantels des Thermoelementkabels.

2, 5 und 8 sind $z u$ den Lötstellen laufende Kabel, bei denen der Bleimantel nicht durchgetrennt worden ist. I, 3, 4, 6, 7, 9 und Io sind Kabel, bei denen die Durchtrennung ausgeführt ist.

Bei der Placierung dieser parallellaufenden Thermoelementkabel wurde eine Vorrichtung ähnlich dem früher (4, s. 114) beschriebenen Hilfsgestell benutzt, in der Furchen für drei parallele Hilfsstäbe so angebracht waren, dass sie alle gleichzeitig in den Erdboden eingeschlagen werden konnten und die Lötstellen der Thermoelemente in bestimmten Abstand voneinander zu liegen kamen. Die Kabel wurden hinter der Schnittstelle hervor umgebogen, am Rand der Arbeitsgrube entlang aufwärts geleitet und in einem etwa $10 \mathrm{~cm}$ tief verlegten, mit Kabelmasse gefüllten Kabelkasten zu einem, nach der Beobachtungshütte führenden Kabel vereinigt. Von den 12 Aderpaaren dieses Kabels waren 10 in Gebrauch, denn für die 
Verfolgung der Temperaturschwankungen des Kabelkastens war auch in diesen ein Thermoelement eingelegt. Das von dem Kabelkasten zu der Beobachtungshütte führende Kabel lief ebenfalls durchgehend in ca. $10 \mathrm{~cm}$ Tiefe hin.

Die Beobachtungen der Versuchsserie wurden etwa einmal in der Woche angestellt. Im folgenden werden einige von ihnen wiedergegeben. Der von der Wärmeübertragung längs des Bleikabels herrührende Fehler war im Herbst und Frühjahr recht klein, wenn in den verschiedenen Messtiefen ungefähr die gleiche Temperatur herrschte (Tabelle 3).

Tabelle 3. Die Temperaturen $\left({ }^{\circ} \mathrm{C}\right)$ an den verschiedenen Messpunkten der Versuchsserie mit abgeschnittenem Bleimantel und der von der Wärmeübertragung

herrührende Fehler.

\begin{tabular}{|c|c|c|c|c|c|c|c|}
\hline \multirow{2}{*}{$\begin{array}{c}\text { Tiefe } \\
\mathrm{cm}\end{array}$} & \multirow{2}{*}{ Nummer } & Temperatur & Fehler & Temperatur & Fehler & Temperatur & Fehler \\
\hline & & \multicolumn{2}{|c|}{ 10.9.1941 um $7 \mathrm{Uhr}$} & \multicolumn{2}{|c|}{ 24. 9. $1941 \mathrm{um} 7 \mathrm{Uhr}$} & \multicolumn{2}{|c|}{ 24. 4. $1941 \mathrm{um} 21 \mathrm{Uhr}$} \\
\hline 10 & 10 & 9.35 & & 8.35 & & -0.15 & \\
\hline 30 & 9 & 11.05 & & 9.65 & & -0.70 & \\
\hline$\Rightarrow$ & 8 & 11.00 & 0.05 & 9.60 & 0.03 & -0.70 & 0.00 \\
\hline ") & 7 & 11.05 & & 9.60 & & -0.70 & \\
\hline 60 & 6 & 11.35 & & 10.05 & & -0.55 & \\
\hline$D$ & 5 & 11.30 & 0.05 & 10.05 & 0.00 & -0.65 & 0.13 \\
\hline " & 4 & 11.35 & & 10.05 & & -0.50 & \\
\hline 90 & 3 & 11.40 & & 10.10 & & 0.00 & \\
\hline i) & 2 & 11.35 & 0.05 & 10.10 & 0.00 & -0.10 & 0.08 \\
\hline , & 1 & 11.40 & & 10.10 & & -0.05 & \\
\hline
\end{tabular}

In der ersten und zweiten Serie, wo die Temperatur des Bodens gleichmässig mit der Tiefe zugenommen hat, ist der Fehler entsprechend verkleinert. In der dritten Serie dagegen ist er am grössten in $60 \mathrm{~cm}$ Tiefe, in der die Temperatur an den Lötstelle der Thermoelemente des unbeschnittenen Kabels niedriger als an den beschnittenen, aber doch an der Verbindungsstelle der Kabel höher ist. Das beruht offenbar darauf, dass die Kabel auf gewissen Strecken durch eine kältere Bodenschicht laufen. Der Fehler ist in den erwähnten Serien unterhalb $0.1^{\circ}$ geblieben.

Während der eigentlichen Sommermonate war der von der Wärmeübertragung herrührende Fehler schon deutlich zu erkennen, wie die Beobachtungen im Juli zeigen (Tabelle 4). 
Tabelle 4. Die Temperaturen $\left({ }^{\circ} \mathrm{C}\right)$ an den verschiedenen Messpunkten der Versuchsserie mit abgeschnittenem Bleimantel und der von der Wärmeübertragung herrührende Fehler.

\begin{tabular}{|c|c|c|c|c|c|c|c|}
\hline \multirow{2}{*}{$\begin{array}{c}\text { Tiefe } \\
\mathrm{cm}\end{array}$} & \multirow{2}{*}{ Nummer } & Temperatur & Fehler & Temperatur & Fehler & Temperatur & Fehler \\
\hline & & \multicolumn{2}{|c|}{ 2. $7.1941 \mathrm{um} 21 \mathrm{Uhr}$} & \multicolumn{2}{|c|}{ 9. $7.1941 \mathrm{um} 21 \mathrm{Uhr}$} & \multicolumn{2}{|c|}{ 30. $7.1941 \mathrm{um} 21 \mathrm{Uhr}$} \\
\hline 10 & 10 & 21.15 & & 23.20 & & 20.95 & \\
\hline 30 & 9 & 18.50 & & 20.40 & & 18.10 & \\
\hline 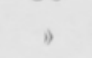 & 8 & 18.95 & 0.45 & 20.90 & 0.53 & 18.85 & 0.75 \\
\hline , & 7 & 18.50 & & 20.35 & & 18.10 & \\
\hline 60 & 6 & 16.90 & & 18.75 & & 12.75 & \\
\hline , & 5 & 17.30 & 0.43 & 19.05 & 0.30 & 13.55 & 0.82 \\
\hline$"$ & 4 & 16.85 & & 18.75 & & 12.70 & \\
\hline 90 & 3 & 15.70 & & 17.60 & & 12.25 & \\
\hline 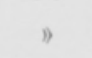 & 2 & 16.05 & 0.28 & 17.85 & 0.18 & 12.70 & 0.47 \\
\hline , & 1 & 15.85 & & 17.75 & & 12.40 & \\
\hline
\end{tabular}

Die Grösse der Fehler zeigt schon deutlich, dass die Abtrennung des Bleimantels notwendig ist. Doch kann der Fehler z.B. im Frühjahr und im Anfang des Sommers, wenn die Temperatur der oberen Bodenschichten noch mehr von den Temperaturen der tieferen Schichten abweicht, wesentlich höher als oben erwähnt ansteigen (Tabelle 5.)

Tabelle 5. Die Temperaturen $\left({ }^{\circ} \mathrm{C}\right)$ an den verschiedenen Messpunkten der Versuchsserie mit abgeschnittenem Bleimantel und der von der Wärmeübertragung herrührende Fehler.

\begin{tabular}{|c|c|c|c|c|c|c|c|}
\hline \multirow{2}{*}{$\begin{array}{l}\text { Tiefe } \\
\mathrm{cm}\end{array}$} & \multirow{2}{*}{ Nummer } & Temperatur & Fehler & Temperatur & Fehler & Temperatur & Fehler \\
\hline & & \multicolumn{2}{|c|}{ 18.6. $1941 \mathrm{um} 21 \mathrm{Uhr}$} & \multicolumn{2}{|c|}{ 21.5.1941 um $21 \mathrm{Uhr}$} & \multicolumn{2}{|c|}{ 28.5. 1941 um $21 \mathrm{Uhr}$} \\
\hline 10 & 10 & 16.85 & & 8.30 & & 15.60 & \\
\hline 30 & 9 & 10.60 & & 2.65 & & 7.85 & \\
\hline 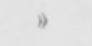 & 8 & 11.55 & 0.95 & 3.65 & 1.00 & 9.05 & 1.15 \\
\hline 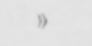 & 7 & 10.60 & & 2.65 & & 7.95 & \\
\hline 60 & 6 & 7.25 & & -0.45 & & 3.35 & \\
\hline$"$ & 5 & 7.85 & 0.60 & 0.10 & 0.55 & 4.35 & 0.98 \\
\hline b & 4 & 7.25 & & -0.45 & & 3.40 & \\
\hline 90 & 3 & 5.35 & & 0.00 & & 1.50 & \\
\hline " & 2 & 5.85 & 0.45 & -0.10 & 0.08 & 1.85 & 0.30 \\
\hline$B$ & 1 & 5.45 & & -0.05 & & 1.60 & \\
\hline
\end{tabular}

Der Fehler ist augenscheinlich desto kleiner, je weiter das unbeschnittene Kabel in Bodenschichten hingelaufen ist, deren Temperatur sich der der Messpunkte nähert. 
Der grösste Fehler wurde bei der Beobachtung am 23. 1. 1941 konstatiert (Tabelle 6).

Tabelle 6. Die Temperaturen $\left({ }^{\circ} \mathrm{C}\right)$ an den verschiedenen Messpunkten der Versuchsserie mit abgeschnittenem Bleimantel und der von der Wärmeübertragung herrührende Fehler am 23. I. I94I.

\begin{tabular}{|c|r|r|r|}
\hline $\begin{array}{c}\text { Tiefe } \\
\mathrm{cm}\end{array}$ & Nummer & Temperatur & Fehler \\
\hline 10 & 10 & -10.65 & \\
30 & 9 & -2.50 & \\
$"$ & 8 & -3.90 & 1.45 \\
, & 7 & -2.40 & \\
60 & 6 & 0.30 & \\
, & 5 & 0.05 & 0.35 \\
, & 4 & 0.50 & \\
90 & 3 & 1.50 & \\
, & 2 & 1.15 & 0.35 \\
, & 1 & 1.50 & \\
\hline
\end{tabular}

Wie man aus den oben angeführten Ergebnissen schliessen kann, genügt der Umstand, dass jedes Thermoelementkabel waagerecht vom Rand der Arbeitsgrube bis zu dem $85 \mathrm{~cm}$ entfernt liegenden Messpunkt läuft, keineswegs dazu, den von der Wärmeübertragung längs des Bleikabels herrührenden Fehler zu eliminieren, sondern die Abtrennung des Bleimantels ist eine notwendige Massnahme gewesen. Wäre sie nicht erfolgt, so hätte das Kabel von dem Messpunkt waagerecht mindestens $1.5-2.0 \mathrm{~m}$ weit verlegt werden müssen, damit der Wärmeübertragungsfehler unterhalb $0.1^{\circ}$ geblieben wäre. Bei der Durchmusterung der oben mitgeteilten Ergebnisse der Versuchsserie mit abgeschnittenem Bleimantel findet man ausserdem, dass die in derselben Tiefe, $20 \mathrm{~cm}$ voneinander entfernt liegenden Lötställen, zu denen Kabel mit abgeschnittenen Bleimanteln laufen, nicht ganz dieselbe Temperatur anzeigen. Der Unterschied kann von der Inhomogenität des Bodens oder davon herrühren, dass es nicht gelungen ist, die Messpunkte in genau dieselbe Tiefe zu verlegen, oder aber er kann auf der Verschiedenheit der Abtrennungsstellen des Bleimantels selbst beruhen. Falls der gefundene Unterschied durch den letztgenannten Umstand bedingt ist, verschwindet sein Einfluss bei den eigentlichen Messungen im Backas, da die einzelnen Kabel von dem Messpunkt an ca. $2.35 \mathrm{~m}$ weit im gleichen Abstand von der Erdoberfläche hinlaufen. 
Wie aus dem Vorstehenden zu ersehen war, können bei der Verwendung von Bleikabeln, ohne den Bleimantel abzutrennen, erhebliche Fehler verursacht werden, die u.a. geeignet sind die Temperaturschwankungen auszugleichen, denn indem in den obersten Bodenschichten z. B. ein Temperaturminimum vorherrscht, kann gleichzeitig in tieferen Schichten noch ein Temperaturmaximum festgestellt werden (vgl. 3, S. 128).

\section{SELOST US.}

MAAN LÄMPÖTILAN MITTAAMISESTA TERMOELEMENTEILLÄ.

Taneli JuUsela.

Yliopiston kasvinviljelystieteellinen laitos, Helsinki.

Helsingin pitäjässä Backaksen tilalla 1. 6. $1940-25.8$. 1942. suoritettujen havaintojen yhteydessä mitattiin maan lämpötila termoelementtien avulla kompensaatiomenetelmää käyttäen. Artikkelissa esitetään mittauskojeisto, sen vakauksen suoritus sekä eräitä mittaustuloksia ynnä ne varokeinot, jotka ovat välttämättömiä lyijyvaippaisia kaapeleita käytettäessä.

\section{LITERATUR.}

(1) Herr, Ludwig. Bodentemperaturen unter besonderer Berücksichtigung der äusseren meteorologischen Faktoren. Berlin 1936.

(2) Heuser, Otto. Untersuchungen über den Temperaturverlauf im Ackerboden. Zeitschrift für Pflanzenernährung, Düngung und Bodenkunde 9, 1930. S. $552-567$.

(3) Juusela, Taneli 1945. Untersuchungen über den Einfluss des Entwässerungsverfahrens auf den Wassergehalt des Bodens, den Bodenfrost und die Bodentemperatur. Acta Agralia Fennica 59, 1945.

(4) Keränen, J. Über die Temperatur des Bodens und der Schneedecke in Sodankylä nach Beobachtungen mit Thermoelementen. Helsingfors, 1920.

(5) Kühl, Wilhelm. Der jährliche Gang der Bodentemperatur in verschiedenen Klimaten. Würzburg 1907 .

(6) Schmidt, Wilhelm. Ein neues Verfahren zur Messung der Bodentemperatur. Zeitschrift für Instrumentenkunde, 46, 1926. S. 431-433.

(7) Semb, Gunnar. Noen undersøkelser over jordtemperaturen og forhold som har innflytelse på den. Meldinger fra Norges Landbrukshøgskole, 18, 1938. S. $1-39$.

(8) Tamm, Ernst. Vergleichende Temperaturmessungen in der Zone des Pflanzenklimas. Landwirtschaftl. Jahrbücher, 83, 1936. S. 457-554.

(9) VDI-Temperaturmessregeln. Hrsg. vom Verein deutscher Ingenieure. 2. Aufl. Berlin. 1940.

(10) Vesikivi, Antri. Suomaan lämpötilamittausten tuloksia. Wissenschaftl. Veröffentl. des Finn. Moorkulturvereins, 15. 1933. 